\title{
Investigating Ternary Li-Mg-Si Zintl Phase Formation and Evolution for Si anodes in Li-ion Batteries with Mg(TFSI)2 Electrolyte Additive
}

\author{
Xiang Li, ${ }^{1 *}$ James A. Gilbert, ${ }^{1}$ Stephen E. Trask, ${ }^{1}$, Ritesh Uppuluri ${ }^{1}$, Saul H. Lapidus ${ }^{2}$, \\ Saida Cora,${ }^{3}$ Niya Sa,${ }^{3}$ Zhenzhen Yang, ${ }^{1}$ Ira D. Bloom, ${ }^{1}$ Fulya Dogan, ${ }^{1}$ John T. Vaughey, \\ ${ }^{1}$, Baris Key, ${ }^{1 *}$ \\ ${ }^{1}$ Chemical Sciences and Engineering Division, Argonne National Laboratory, Lemont, IL \\ 60439, United States. \\ ${ }^{2}$ Advanced Photon Source, Argonne National Laboratory, Lemont, IL 60439, United States. \\ ${ }^{3}$ Chemistry Department, University of Massachusetts, Boston, Massachusetts 02125, \\ United States
}




\section{Supporting Information:}

(a)

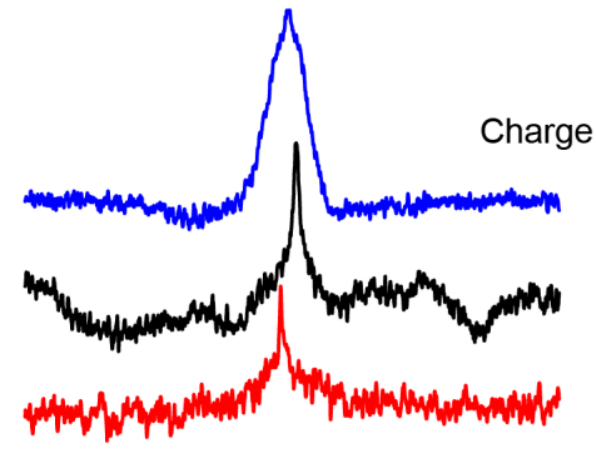

(b)

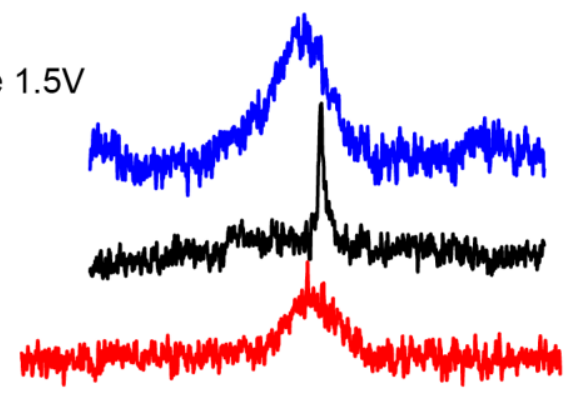

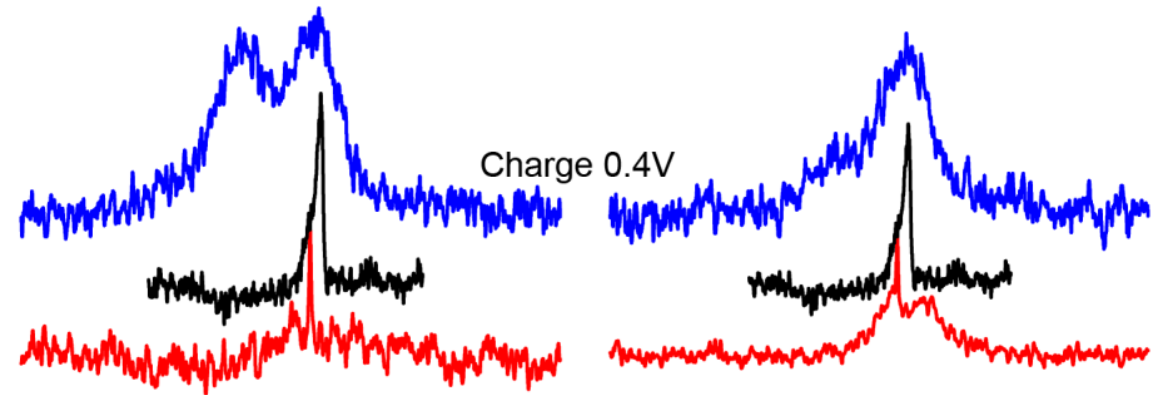

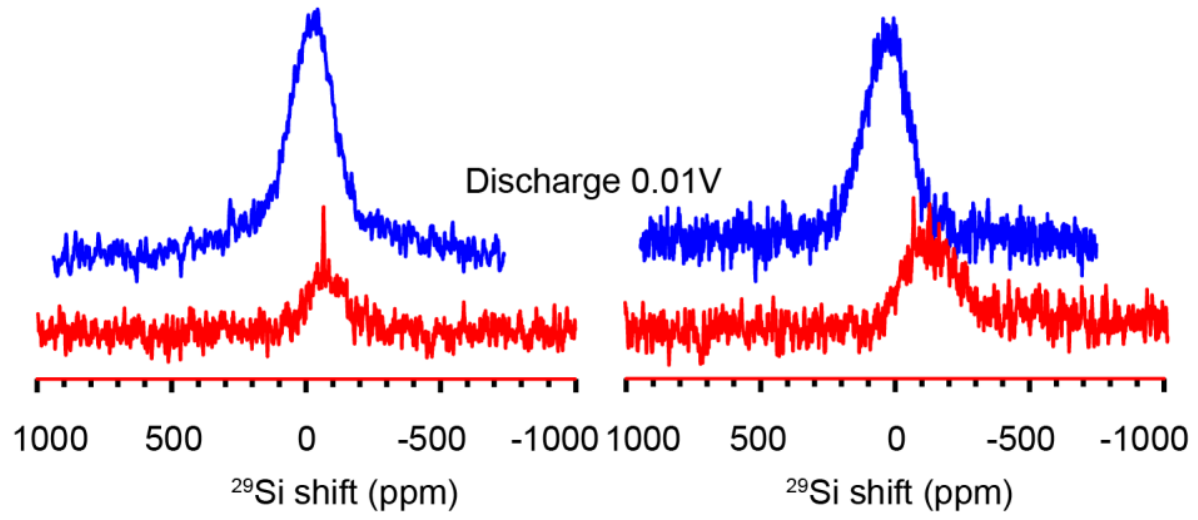

Figure S1. One pulse, Hahn echo, and Li-Si CP NMR on GFM(a) and GF(b) at $10 \mathrm{mV}$, $400 \mathrm{mV}$, and $1500 \mathrm{mV}$.
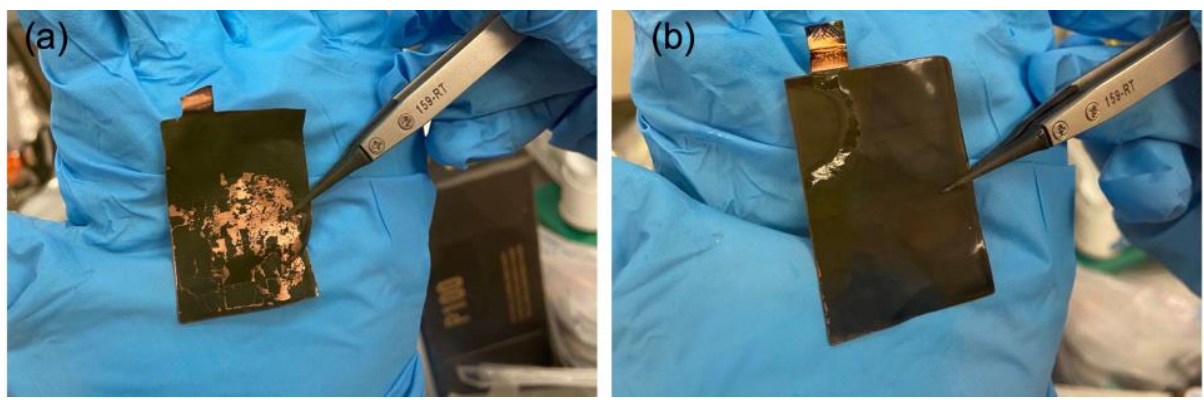
Figure S2. Delamination comparison after 270 cycles between GF (a) and GFM (b) in full cell testing. (detailed results are beyond the scope of this work and will be presented in a separate study) The electrodes are the same as those used in pouch half cells but with different sizes, so it's representative of the level of delamination.

(a)

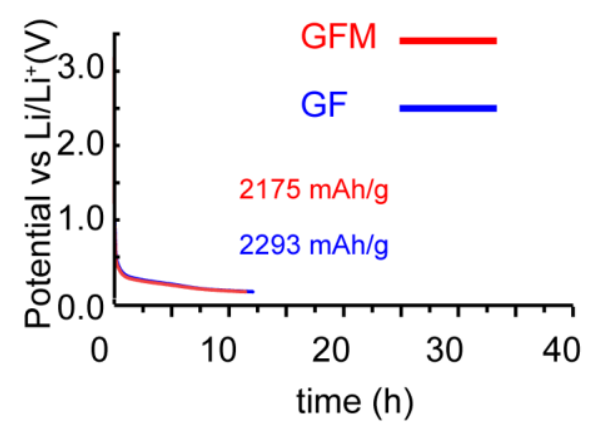

(c)

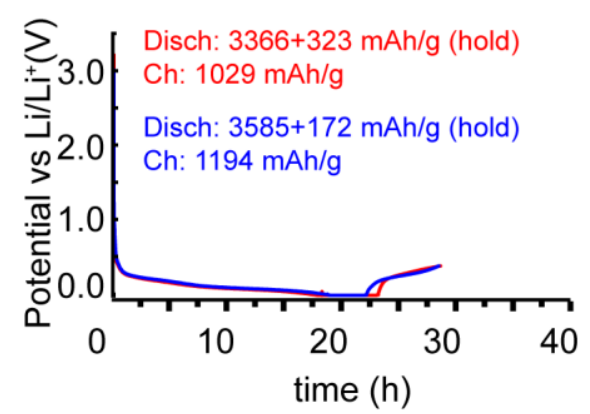

(b)

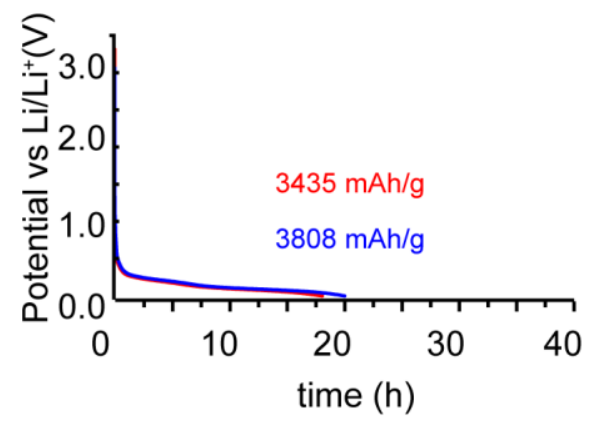

(d)

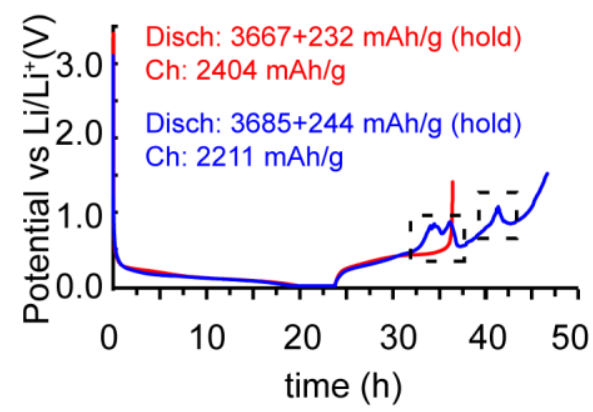

Figure S3. Electrochemical profiles of GF and GFM pouch cells. At the fully delithiated state, the GF cell exhibits abnormal fluctuating charge curve. Given that all the half cells were cycled without additional pressure. It is possibly due to severe delamination of the Si electrode, especially when some of the lithiated Si particles occasionally detach from current collector and obtain contact again. 

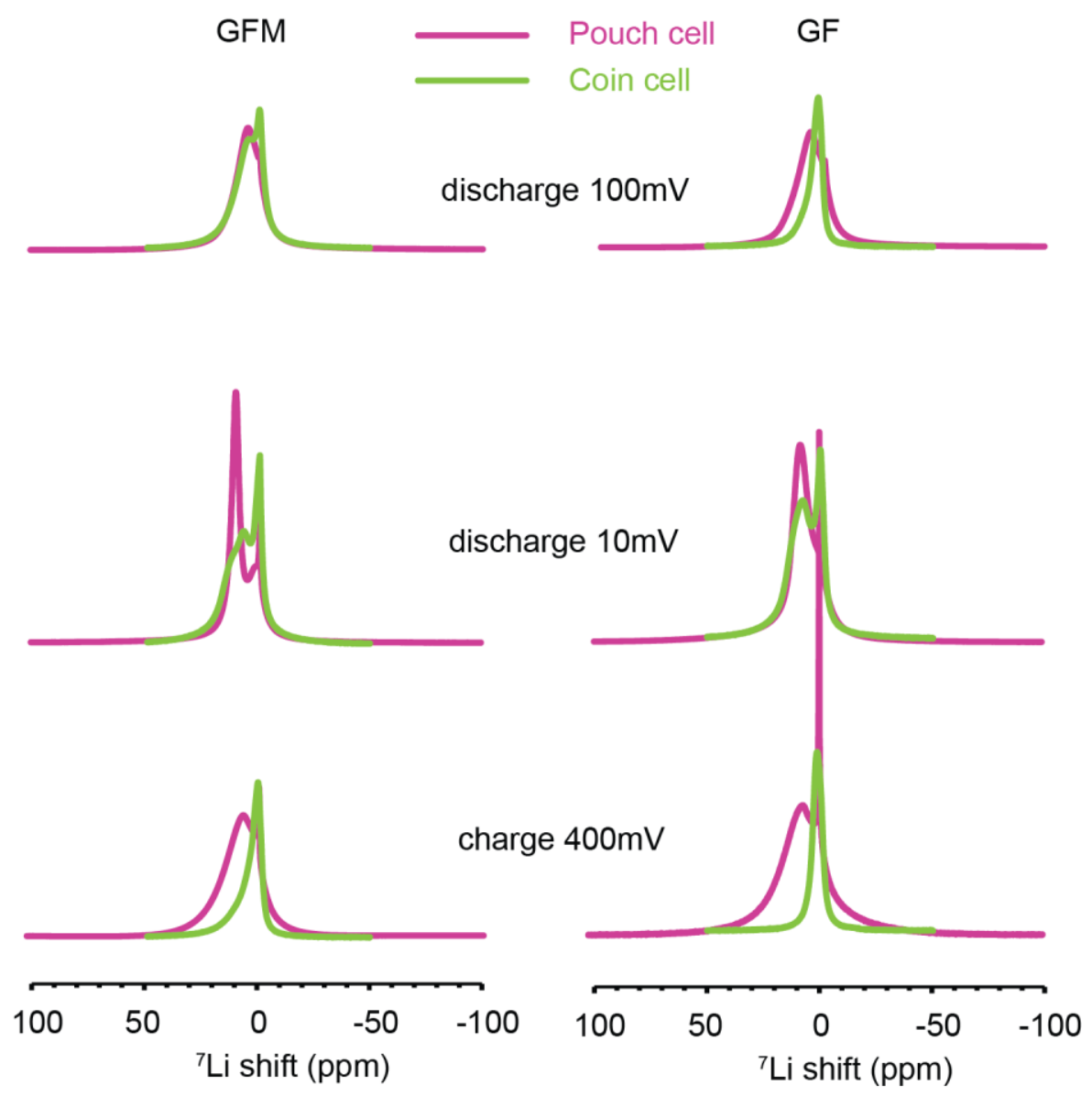

Figure S4. ${ }^{7} \mathrm{Li}$ NMR spectra comparison between pouch cells(purple) and coin cells(yellow) for GFM and GF.

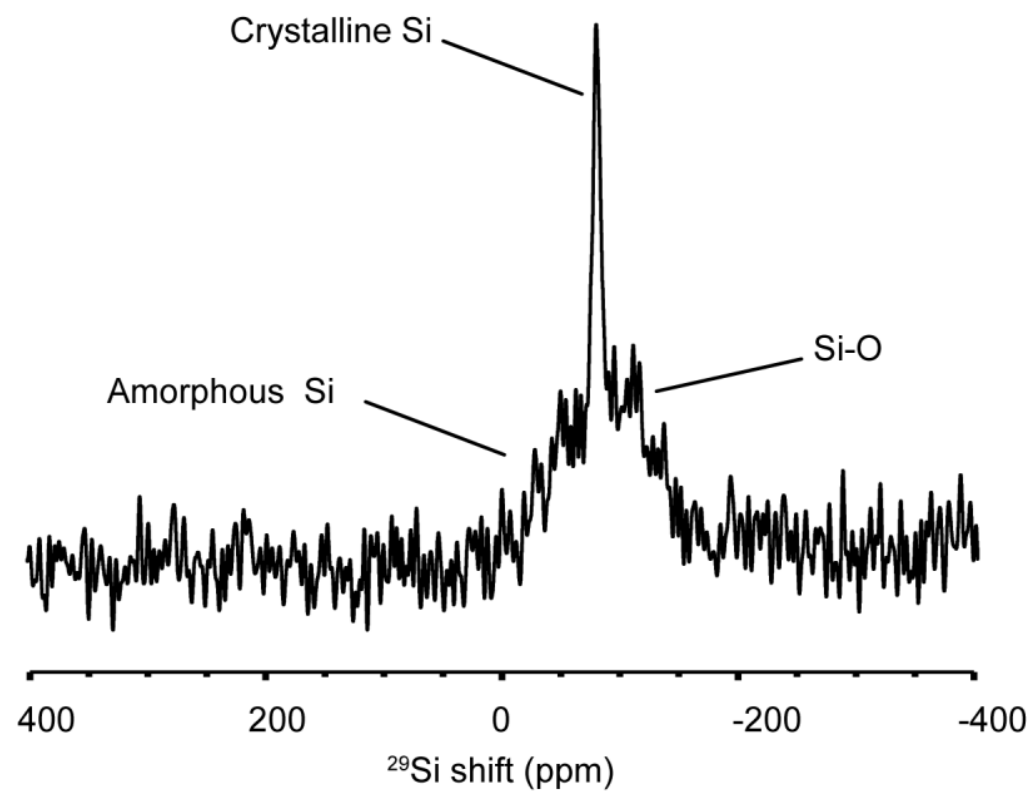


Figure S5. ${ }^{29} \mathrm{Si}$ NMR spectrum of pristine Si powder.

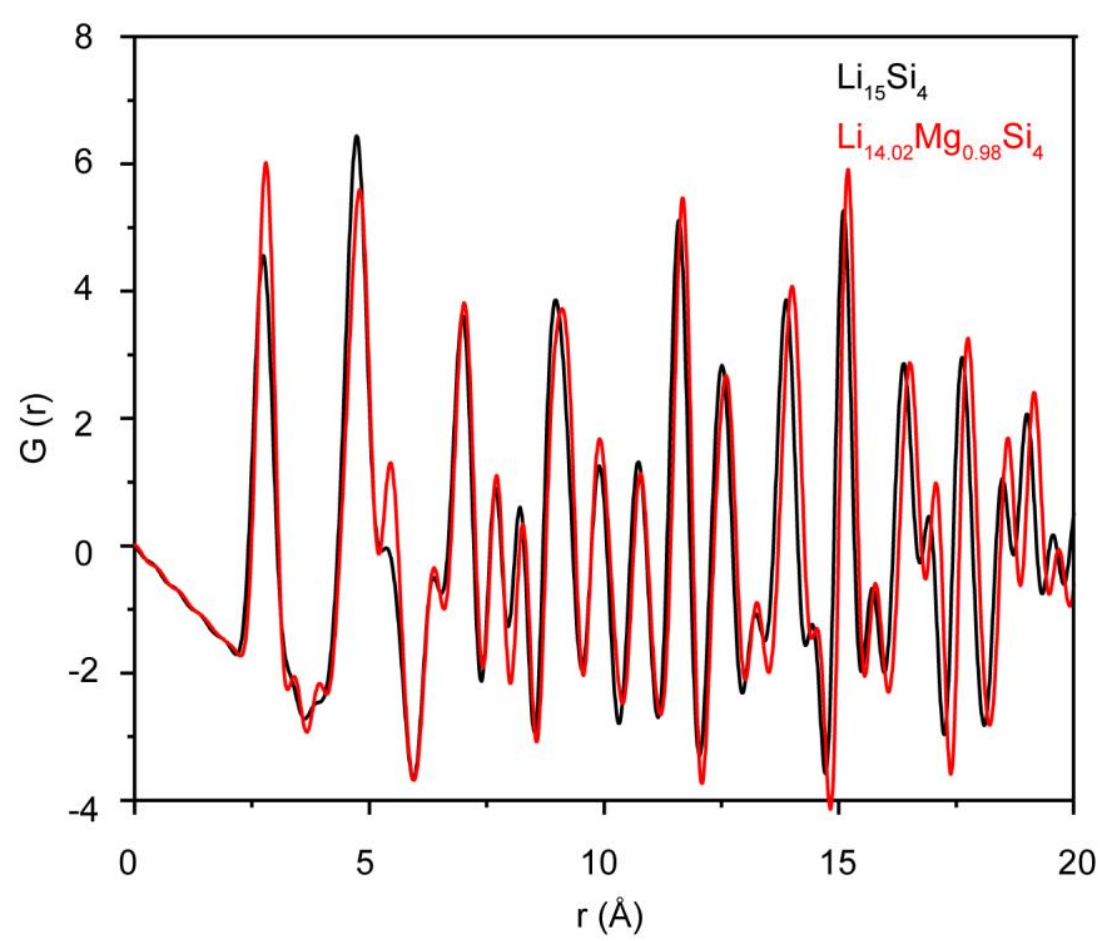

Figure S6. Calculated PDFs of $\mathrm{Li}_{15} \mathrm{Si}_{4}$ and $\mathrm{Li}_{14.02} \mathrm{Mg}_{0.98} \mathrm{Si}_{4}$ structure models.
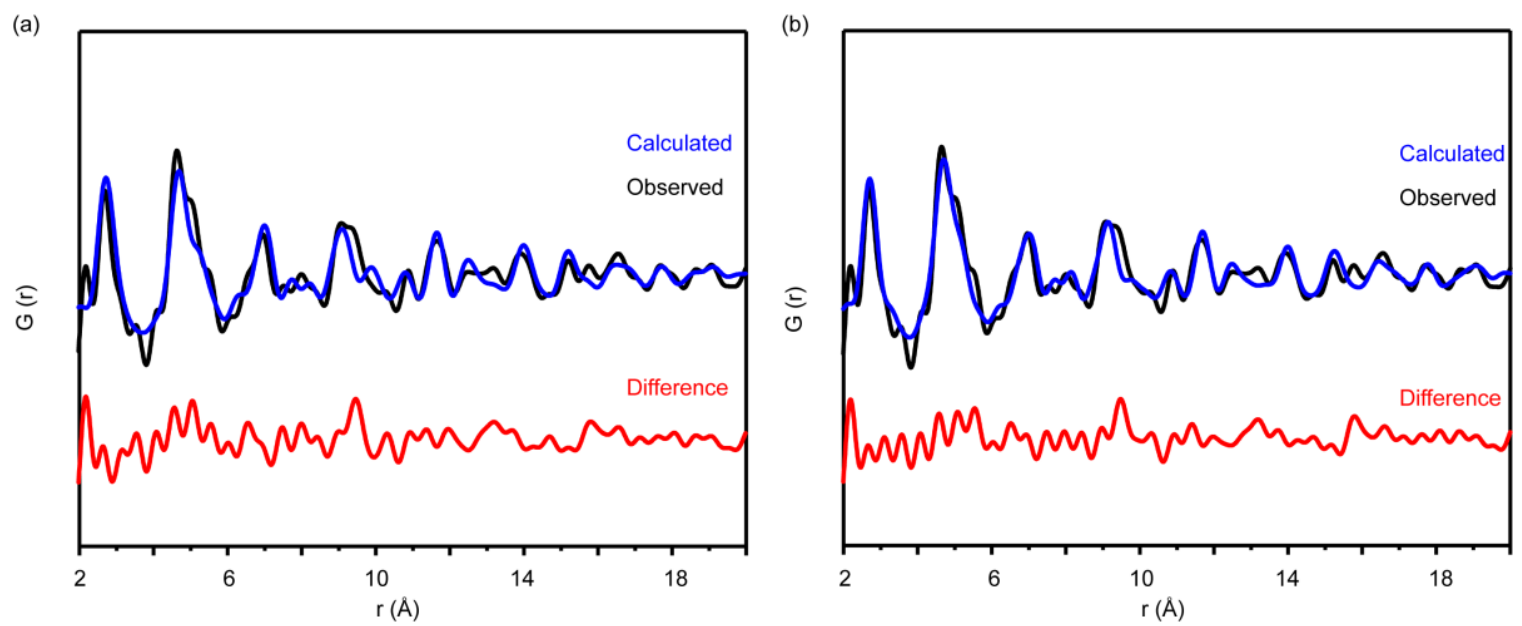

Figure S7. PDF fit of silicon anode discharged to $10 \mathrm{mV}$ against $\mathrm{Li}_{14.02} \mathrm{Mg}_{0.98} \mathrm{Si}_{4}$ (a) and $\mathrm{Li}_{15} \mathrm{Si}_{4}$ (b) model in GFM electrolyte.

Table S1. ${ }^{7} \mathrm{Li}$ NMR spectra deconvolution parameters of GF coin cells. 


\begin{tabular}{|c|c|c|c|c|}
\hline & shift/ppm & $\mathrm{LB} / \mathrm{Hz}$ & $x G /(1-x) L$ & $\%$ \\
\hline $\begin{array}{c}1 \mathrm{st} \text { dis } \\
0.2 \mathrm{~V}\end{array}$ & -0.92 & 500 & 0.2 & 0.588 \\
\hline & 0.22 & 270 & 0.3 & 0.284 \\
\hline & 1.4 & 500 & 0.5 & 0.128 \\
\hline $0.15 \mathrm{~V}$ & -0.75 & 500 & 0.2 & 0.288 \\
\hline & 0.4 & 450 & 0.8 & 0.171 \\
\hline & 2.2 & 700 & 0.1 & 0.541 \\
\hline $0.1 \mathrm{~V}$ & -0.2 & 650 & 0.2 & 0.419 \\
\hline & 1.7 & 600 & 0.3 & 0.445 \\
\hline & 4.25 & 1000 & 0.2 & 0.136 \\
\hline $0.01 \mathrm{~V}$ & -0.4 & 500 & 0.1 & 0.197 \\
\hline & 1.7 & 600 & 0.1 & 0.065 \\
\hline & 7.5 & 2000 & 0.45 & 0.567 \\
\hline & 12.8 & 2000 & 0.1 & 0.171 \\
\hline 1st ch $0.4 \mathrm{~V}$ & 0.2 & 640 & 0.5 & 0.429 \\
\hline & 2.4 & 600 & 0.3 & 0.178 \\
\hline & 5 & 1600 & 0.3 & 0.337 \\
\hline & 12.5 & 2000 & 0.1 & 0.056 \\
\hline $\begin{array}{l}\text { 2nd dis } \\
0.01 \mathrm{~V}\end{array}$ & -0.1 & 400 & 0.2 & 0.194 \\
\hline & 1.4 & 600 & 0.3 & 0.104 \\
\hline & 5.1 & 1700 & 0.23 & 0.573 \\
\hline & 13.3 & 2000 & 0.5 & 0.228 \\
\hline & -1.6 & 400 & 0.2 & 0.095 \\
\hline
\end{tabular}

Table S2. ${ }^{7} \mathrm{Li}$ NMR spectra deconvolution parameters of GFM coin cells 


\begin{tabular}{|c|c|c|c|c|}
\hline & shift/ppm & $\mathrm{LB} / \mathrm{Hz}$ & $x G /(1-2) L$ & $\%$ \\
\hline \multirow{2}{*}{$\begin{array}{l}1 \text { st dis } \\
0.35 \mathrm{~V}\end{array}$} & -0.98 & 110 & 0.4 & 0.202 \\
\hline & -1.2 & 500 & 0.1 & 0.798 \\
\hline \multirow[t]{2}{*}{$0.2 \mathrm{~V}$} & -0.15 & 600 & 0.5 & 0.921 \\
\hline & 1.5 & 1000 & 0.5 & 0.079 \\
\hline \multirow[t]{2}{*}{$0.15 \mathrm{~V}$} & 0.55 & 850 & 0.1 & 0.465 \\
\hline & 1.7 & 1500 & 0.1 & 0.535 \\
\hline \multirow[t]{4}{*}{$0.1 \mathrm{~V}$} & -0.95 & 500 & 0.2 & 0.133 \\
\hline & 16 & 2500 & 0.1 & 0.095 \\
\hline & 4.25 & 2400 & 0.6 & 0.759 \\
\hline & 1.4 & 500 & 0.5 & 0.013 \\
\hline \multirow[t]{4}{*}{$0.05 \mathrm{~V}$} & -0.3 & 400 & 0.5 & 0.165 \\
\hline & 4.7 & 2000 & 0.3 & 0.535 \\
\hline & 15 & 2500 & 0.3 & 0.228 \\
\hline & 1.6 & 500 & 0.5 & 0.072 \\
\hline \multirow[t]{5}{*}{$0.01 \mathrm{~V}$} & -0.5 & 400 & 0.1 & 0.145 \\
\hline & 1.2 & 600 & 0.1 & 0.116 \\
\hline & 6.2 & 1650 & 0.1 & 0.387 \\
\hline & 12.5 & 2000 & 0.1 & 0.322 \\
\hline & 25 & 2500 & 0.2 & 0.03 \\
\hline \multirow{3}{*}{$\begin{array}{l}1 \mathrm{st} \mathrm{ch} \\
0.2 \mathrm{~V}\end{array}$} & -0.4 & 300 & 0.3 & 0.133 \\
\hline & 1 & 600 & 0.2 & 0.244 \\
\hline & 5 & 1600 & 0.2 & 0.395 \\
\hline
\end{tabular}




\begin{tabular}{|c|c|c|c|c|}
\hline & 11 & 2000 & 0.5 & 0.207 \\
\hline & 25 & 2500 & 0.5 & 0.022 \\
\hline \multirow{5}{*}{$0.3 \mathrm{~V}$} & -0.85 & 300 & 0.3 & 0.045 \\
\hline & 0.6 & 550 & 0.1 & 0.197 \\
\hline & 4.7 & 2000 & 0.7 & 0.485 \\
\hline & 12.6 & 2000 & 0.2 & 0.242 \\
\hline & 25 & 2500 & 0.5 & 0.03 \\
\hline \multirow[t]{5}{*}{$0.4 \mathrm{~V}$} & -0.15 & 550 & 0.6 & 0.243 \\
\hline & 1.8 & 700 & 0.4 & 0.172 \\
\hline & 4.2 & 2000 & 0.6 & 0.426 \\
\hline & 12.5 & 2000 & 0.1 & 0.117 \\
\hline & 22 & 2500 & 0.1 & 0.042 \\
\hline \multirow[t]{4}{*}{$1.5 \mathrm{~V}$} & -0.7 & 360 & 0.1 & 0.415 \\
\hline & 0.58 & 370 & 0.15 & 0.352 \\
\hline & 4.2 & 1500 & 0.4 & 0.053 \\
\hline & 1.5 & 700 & 0.7 & 0.18 \\
\hline \multirow[t]{5}{*}{$\begin{array}{c}\text { 2nd dis } \\
0.1 \mathrm{~V}\end{array}$} & -0.9 & 500 & 0.3 & 0.166 \\
\hline & 0.2 & 330 & 0.15 & 0.116 \\
\hline & 1.6 & 600 & 0.35 & 0.286 \\
\hline & 4.7 & 1500 & 0.3 & 0.344 \\
\hline & 11.5 & 2000 & 0.3 & 0.088 \\
\hline \multirow[t]{4}{*}{ dis $0.01 \mathrm{~V}$} & -0.1 & 400 & 0.1 & 0.1 \\
\hline & 1.3 & 650 & 0.1 & 0.115 \\
\hline & 5.8 & 1700 & 0.3 & 0.314 \\
\hline & 13.7 & 2000 & 0.5 & 0.283 \\
\hline
\end{tabular}




\begin{tabular}{|c|c|c|c|c|}
\hline & 28.5 & 3500 & 0.15 & 0.05 \\
\hline & -1.5 & 400 & 0.2 & 0.05 \\
\hline & -6 & 2500 & 0.3 & 0.087 \\
\hline
\end{tabular}

Table S3. ${ }^{7} \mathrm{Li}$ NMR spectra deconvolution parameters of GF pouch cells

\begin{tabular}{|c|c|c|c|c|}
\hline & Shift/ppm & LB/Hz & $\mathrm{xG} /(1-\mathrm{x}) \mathrm{L}$ & $\%$ \\
\hline $\mathrm{dis} 100$ & 5.2 & 1695 & 0.46 & 0.979 \\
\hline $\mathrm{mV}$ & -1.2 & 205 & 0.64 & 0.021 \\
\hline & & & & \\
\hline & 8.2 & 1000 & 0.5 & 0.664 \\
\hline $10 \mathrm{mV}$ & -0.5 & 1000 & 0.2 & 0.241 \\
\hline & -1.4 & 100 & 0.2 & 0.004 \\
\hline & 22 & 2500 & 0.2 & 0.091 \\
\hline & & & & \\
\hline & 6.4 & 2228 & 0.3 & 0.855 \\
\hline char 400 & -2 & 1000 & 0.1 & 0.084 \\
\hline $\mathrm{mV}$ & -1.72 & 50 & 0.5 & 0.061 \\
\hline & & & & \\
\hline & 10.6 & 1900 & 0.3 & 0.713 \\
\hline & -0.6 & 1215 & 0.1 & 0.274 \\
\hline & -1.72 & 45 & 8 & 0.013 \\
\hline $1500 \mathrm{mV}$ & & & & \\
\hline
\end{tabular}

Table S4. ${ }^{7} \mathrm{Li}$ NMR spectra deconvolution parameters of GFM pouch cells

\begin{tabular}{|c|c|c|c|c|}
\hline & shift & LB & $\mathrm{x}$ & $\%$ \\
\hline $100 \mathrm{mV}$ & 4 & 1400 & 0.35 & 0.979 \\
\hline & -1.37 & 200 & 0.6 & 0.021 \\
\hline & & & & \\
\hline $10 \mathrm{mV}$ & 9 & 460 & 0.41 & 0.529 \\
\hline & 13 & 1300 & 0.29 & 0.106 \\
\hline
\end{tabular}




\begin{tabular}{|c|c|c|c|c|}
\hline & 0.3 & 1020 & 0.33 & 0.334 \\
\hline & -1.8 & 65 & 0.11 & 0.031 \\
\hline & & & & \\
\hline 400 & 4.97 & 1686 & 0.84 & 0.577 \\
\hline & 15.81 & 2316 & 0.54 & 0.247 \\
\hline & -1.4 & 98 & 0.13 & 0.03 \\
\hline & 0.173 & 2177 & 0.23 & 0.146 \\
\hline & & & & \\
\hline 1500 & 3.3 & 1000 & 0.21 & 0.763 \\
\hline & -1.75 & 50 & 0.13 & 0.055 \\
\hline & 13 & 1300 & 0.33 & 0.086 \\
\hline & -3 & 800 & 0.28 & 0.097 \\
\hline
\end{tabular}

Table S5. ${ }^{29}$ Si NMR spectra deconvolution parameters of GF pouch cells.

\begin{tabular}{|c|c|c|c|c|c|c|}
\hline & shift/ppm & CSA & $\eta$ & LB/kHz & GB/kHz & $\%$ \\
\hline $\begin{array}{c}\text { dis 100 } \\
\mathrm{mV}\end{array}$ & -10 & 70 & 0.8 & 11000 & 4000 & 0.861 \\
\hline & 220 & 200 & 0.1 & 5000 & 3000 & 0.107 \\
\hline & -75 & 165 & 0.2 & 700 & 900 & 0.031 \\
\hline & & & & & & \\
\hline $10 \mathrm{mV}$ & 30 & 150 & 0.7 & 10000 & 2000 & 1 \\
\hline & & & & & & \\
\hline $\begin{array}{c}\text { char } 400 \\
\mathrm{mV}\end{array}$ & 180 & 150 & 0.7 & 4000 & 4500 & 0.116 \\
\hline & -70 & 300 & 0.5 & 500 & 500 & 0.009 \\
\hline & -65 & 260 & 0.8 & 13000 & 2000 & 0.875 \\
\hline
\end{tabular}

Table S6. ${ }^{29}$ Si NMR spectra deconvolution parameters of GFM pouch cells.

\begin{tabular}{|c|c|c|c|c|c|c|}
\hline & shift/ppm & CSA & $\eta$ & $\mathrm{LB} / \mathrm{kHz}$ & $\mathrm{GB} / \mathrm{kHz}$ & $\%$ \\
\hline $\begin{array}{c}\text { dis } 100 \\
\mathrm{mV}\end{array}$ & -80 & 80 & 0.2 & 700 & 800 & 0.065 \\
\hline
\end{tabular}




\begin{tabular}{|c|c|c|c|c|c|c|}
\hline & -18 & 280 & 0.7 & 13000 & 2000 & 0.868 \\
\hline & 225 & 140 & 0.2 & 2000 & 800 & 0.066 \\
\hline & & & & & & \\
\hline $10 \mathrm{mV}$ & -25 & 90 & 0.5 & 11500 & 2000 & 1 \\
\hline & & & & & & \\
\hline $\begin{array}{c}\text { char } 400 \\
\mathrm{mV}\end{array}$ & -63 & 110 & 0.2 & 500 & 300 & 0.007 \\
\hline & -72 & 200 & 0.8 & 11000 & 2000 & 0.48 \\
\hline & 180 & 85 & 0.7 & 13000 & 2000 & 0.514 \\
\hline & -90 & 300 & -0.4 & 7000 & 9600 & 0.936 \\
\hline $\begin{array}{c}\text { char } 1500 \\
\mathrm{mV}\end{array}$ & -85 & 300 & 0.4 & 700 & 500 & 0.006 \\
\hline & 140 & 167 & 0.4 & 4000 & 5000 & 0.059 \\
\hline
\end{tabular}

Table S7: Refined parameters for discharged $\mathrm{Si}$ anode against $\mathrm{Li}_{15} \mathrm{Si}_{4}$ and $\mathrm{Li}_{14.02} \mathrm{Mg}_{0.98} \mathrm{Si}_{4}$.

\begin{tabular}{|c|c|c|c|}
\hline Phase & Scale Factor & Delta2 & $\mathrm{a}(\AA)$ \\
\hline $\mathrm{Li}_{15} \mathrm{Si}_{4}$ & 0.4720 & 0.9934 & 10.7231 \\
\hline $\mathrm{Li}_{14.02} \mathrm{Mg}_{0.98} \mathrm{Si}_{4}$ & 0.4627 & 0.9504 & 10.7095 \\
\hline
\end{tabular}

